\title{
Protocolo do perfil das habilidades de comunicação de crianças surdas
}

\author{
Protocol of Deaf Children Communicative Skills Profile
}

\author{
Felipe Venâncio Barbosa \\ Universidade de São Paulo \\ Ida Lichtig \\ Universidade de São Paulo
}

\begin{abstract}
Resumo
A avaliação da comunicação de crianças surdas tem sido restringida à modalidade oral. As habilidades comunicativas que se pautam fora dessa modalidade são geralmente negligenciadas e o potencial comunicativo presente nas crianças surdas é desvalorizado, colocando a criança surda em desnível constante em relação ao desempenho das crianças ouvintes. Isso ocorre porque os protocolos de avaliação geralmente não são focados na avaliação de crianças surdas e não privilegiam as particularidades da modalidade visuoespacial em situações de exame. O protocolo "Avaliação das Habilidades Comunicativas e de Linguagem" (LICHTIG, 2004), elaborado e aplicado em crianças de 3 a 6 anos de idade, é pioneiro na avaliação focada na criança surda. Este estudo tem por objetivo apresentar duas propostas de alteração do protocolo elaborado por Lichtig et al. (2004). As modificações apresentadas visam facilitar a análise e agilizar sua aplicação, provendo subsídios suficientes para o delineamento de procedimentos terapêuticos e de intervenção educacional.
\end{abstract}

\section{Palavras-Chave}

Avaliação, língua de sinais, linguagem, audiologia educacional. 


\begin{abstract}
The assessment of deaf children communication has been restricted to their oral production. Communication skills based on non-oral languages are usually neglected and the communicative potential shown by deaf children is therefore underestimated, which leaves deaf children in constant disadvantage in relation to the hearing children performance. The reason for this phenomenon lies on the fact that protocols do not focus on assessing deaf children and do not highlight the visual spatial mode. The "Assessment of Communication Skills and Language" (LICHTIG, 2004), developed in order to be used with children from 3-6 years old, is a pioneer protocol of assessment in this regard. This study aims to present two proposals to alter the protocol developed by Lichtig et al. (2004). The proposals will be presented as to facilitate the protocol application and its analysis, as well as to make the whole process faster. As a result, the proposed changes can support the design of therapeutic procedures and educational intervention.
\end{abstract}

\title{
Keywords
}

Assessment, sign language, language, educational audiology. 


\title{
Introdução
}

\begin{abstract}
A
aquisição de língua e o desenvolvimento de linguagem são influenciados pelos estímulos que a criança recebe do meio (MOGFORD; BISHOP, 2002). Assim como para o desenvolvimento cognitivo adequado, a linguagem necessita de subsídios vindos do input gerado pelos usuários de uma língua, membros da comunidade em que a criança está imersa, além de outros componentes como os psicológicos e os sociais (MOGFORD; BISHOP, 2002), que também participam do processo de aquisição de língua e desenvolvimento de linguagem. A acessibilidade à língua é, então, um princípio para que a própria língua seja adquirida e para que haja desenvolvimento da linguagem e da cognição adequado.

Para a aquisição integral de uma língua de modalidade oral-auditiva é necessário que as habilidades auditivas estejam preservadas (BORGES; SALOMÃO, 2003). São muitos os estudos encontrados na literatura científica que relacionam as perdas auditivas com os insucessos na aquisição de língua e desenvolvimento de linguagem (BALBANI; MANTOVANI, 2003). No caso da criança surda com perda auditiva severa e/ou profunda, o acesso a esta modalidade de língua fica sobremodo comprometido, e isso torna a aquisição da língua oral uma tarefa árdua, não ocorrendo de forma natural (LEVY; BARBOSA, 2005). A melhor e mais coerente forma de estimulação de linguagem para uma criança surda é, portanto, aquela que se baseia na utilização das línguas de sinais como primeira língua, o que não descarta o aprendizado da língua oral (PICKERSGILL, 1998).

As línguas de sinais são línguas naturais de modalidade visuoespacial utilizadas pelas comunidades surdas (FERREIRA-BRITO, 1995; QUADROS, 1997; QUADROS; KARNOPP, 2004). São línguas que não dependem da audição para serem adquiridas, são completas e propiciam integralmente a estruturação das habilidades cognitivas. Por não dependerem da audição, são línguas naturalmente adquiridas pelas pessoas surdas e, por meio delas, as informações são facilmente compreendidas e a expressão das ideias dessas pessoas é veiculada sem impedimentos.
\end{abstract}


A avaliação das habilidades comunicativas de uma criança surda, dessa forma, deve sempre levar em consideração as dificuldades que esta possa ter quanto ao uso da língua oral e observar o uso e o domínio da língua de sinais. Para que isso seja viável, é necessário que o avaliador tenha domínio dessas línguas e assim o processo avaliativo poderá ocorrer de forma eficaz. A possibilidade de viés no resultado da avaliação aplicada por um examinador sem conhecimentos da língua de sinais é uma realidade que não pode ser negligenciada porque o desenvolvimento de linguagem em uma criança surda poderá ocorrer através da aquisição da língua de sinais, que é uma língua cuja modalidade (visuoespacial) não apresenta restrições de acesso, desde que não exista a presença de outros comprometimentos sensoriais associados à surdez. Analisar o desenvolvimento das habilidades comunicativas e de linguagem apenas através da língua oral é inadequado e insuficiente, pois o indivíduo surdo poderá ser erroneamente classificado como portador de um distúrbio de linguagem, sendo que na realidade ele estaria em uma etapa de aprendizado da língua oral não esperada para sua idade quando comparada com a aquisição oral de uma criança ouvinte.

A elaboração de avaliações específicas para averiguar a aquisição e o desenvolvimento da língua de sinais é de extrema necessidade no Brasil, haja vista o reduzido número de pesquisadores empenhados nesta área e a escassez de estudos linguísticos que possam contribuir na estrutura básica do delineamento de um protocolo. Mesmo em estudos descritivos da Língua de Sinais Brasileira, são poucas as tentativas (FERREIRA-BRITO, 1995; QUADROS; KARNOPP, 2004) e os trabalhos já existentes são abrangentes, dificultando a investigação fonoaudiológica em aspectos específicos da língua.

Os protocolos existentes, focados nas habilidades da língua oral, com frequência fornecem achados que são generalizados para as habilidades cognitivas da pessoa surda, muitas vezes testando suas habilidades cognitivas em uma língua de difícil acesso sem considerar a variável "domínio da língua" ou a forma como os estímulos são apresentados. Equívocos como esse podem ser prejudiciais à intervenção terapêutica, pois podem atribuir características de distúrbios de linguagem ou distúrbios cognitivos a crianças surdas normais.

Um questionamento constante na produção dos estudiosos da surdez refere-se à forma como podem ser elaborados os instrumentos de avaliação para a criança surda (LEVY; BARBOSA, 2005; WOLL, 1998).

A tradição oralista dentro da Fonoaudiologia e da área da Educação promoveu a tendência da adaptação de testes elaborados para crianças ouvintes 
às práticas da avaliação e intervenção em surdez. Especificamente, dentro do campo da Audiologia Educacional, área da Fonoaudiologia que lida com os quadros de surdez, os protocolos de avaliação elaborados em sua maioria tendem à avaliação de aspectos da oralidade.

Alpiner e McCarthy (1987) apresentam uma série de protocolos elaborados para a avaliação de pessoas com surdez, entretanto todos se baseiam na investigação de habilidades auditivas ou em habilidades comunicativas com foco no uso da língua oral. Esses autores apresentam uma série de instrumentos utilizados para avaliar a função comunicativa de surdos adultos. Esses instrumentos, apesar de serem elaborados para pessoas surdas, foram construídos com base na língua inglesa e, portanto, são passíveis de adaptaçôes, pelo foco na língua oral e pela negligência habitual das habilidades gerais de linguagem.

Diante da escassez de instrumentos de avaliação, é muito frequente a utilização de testes da língua oral para a língua de sinais, como o Teste de Boston para Diagnóstico das Afasias (KAPLAN et al., 1996), e o Teste de Vocabulário de Boston (KAPLAN et al., 1996), o Token Test (DE RENZI; VIGNOLO, 1962), TIPITI (BRAZ; PELLICCIOTTI, 1981), entre outros. Esse tipo de adaptação precisa ser cuidadoso para que as interferências entre as modalidades de língua (LEVY; BARBOSA, 2005) não provoquem erros tanto na aplicação como na avaliação do teste.

Levy e Barbosa (2005) apresentam algumas provas já descritas na literatura e que podem ser adaptadas para a avaliação da pessoa surda, como o Teste de Vocabulário do ABFW (BEFI-LOPES, 2000), e a Avaliação da Pragmática (FERNANDES, 2000). Para os instrumentos elaborados em uma língua estrangeira, o cuidado com a adaptação deve ser ainda maior, levando em consideração não apenas as características da língua em que o teste foi construído, mas as características da cultura e o contexto de sua elaboração.

Para a elaboração de avaliações na área da surdez e que levem em conta a língua de sinais, são necessárias pesquisas sobre a aquisição e desenvolvimento normais dessa língua. Acrescenta-se que deverão ser consideradas as diversidades quanto à data da aquisição da língua de sinais (GRIMSHAW et al., 1998), ao tipo de estímulo ao qual a criança foi exposta, ao domínio da língua pelos pais (NICHOLAS; GEERS, 2003), ao grau da perda auditiva da criança, à data de diagnóstico e ao tipo de procedimento utilizado, ao seguimento em terapia fonoaudiológica, à abordagem terapêutica à qual a criança foi submetida, entre 
muitas outras variáveis pessoais que influenciarão nos padrões de aquisição de língua e desenvolvimento de linguagem.

A preocupação com a pesquisa de metodologias de avaliação de linguagem da pessoa surda tem motivado diversos estudiosos atualmente (HERMAN et al., 2004; MORGAN, 2006; MORGAN, 2007). As investigações neste campo alcançam cada vez maiores níveis de desenvolvimento dentro do estudo da linguagem (LICHTIG, 2004; ATKINSON et al., 2005; LEVY; BARBOSA, 2005), cognição (CARVALHO, 2005; BARBOSA, 2006; MORGAN, 2006) e em suas aplicações no contexto social, principalmente com as novas propostas para a atuação fonoaudiológica em surdez (LODI, 2000; LEVY; BARBOSA, 2005; ATKINSON et al., 2005).

Herman (1998) iniciou os estudos de metodologias de avaliação da aquisição da Língua de Sinais Britânica do ponto de vista linguístico. Essa autora, com a divulgação de estudos que legitimam as línguas de sinais com o mesmo status que as línguas orais, publicou em 1999 o teste "Assessing British Sign Language Development", que avalia o desenvolvimento da língua de sinais em sua compreensão (HERMAN et al. 1999) e produção (HERMAN et al., 2004), padronizando os procedimentos para serem utilizados posteriormente por clínicos e pesquisadores. Nesta época a carência de instrumentos de avaliação já estava sendo sinalizada, assim como a necessidade de normatização dos testes.

Avaliar o desenvolvimento de linguagem em surdos usuários de línguas de sinais não é apenas uma curiosidade científica. Os distúrbios de linguagem costumeiramente expressos nas línguas orais, sejam primários ou secundários, são também expressos nas línguas de sinais (BARBOSA, 2005; LEVY; BARBOSA, 2005). Por isso, o desenvolvimento de instrumentos de avaliação específicos, inclusive para os quadros em que a atipia da língua de sinais é identificada, são de grande importância e sua falta interfere diretamente na qualidade da intervenção clínica de fonoaudiólogos.

Atkinson et al. (2005) realizaram um estudo com surdos afásicos, criando testes de habilidades de compreensão da Língua de Sinais Britânica. Dentre os testes elaborados pelos autores, destacam-se o "The Sign to Picture Matching Test" $\mathrm{e}$ "Test of verb and sentence comprehension".

Lichtert (2003) apresenta a aplicação do questionário pragmático Protocol for the Assessment of Prelinguistic Intentional Communication (PAPIC) elaborado por Casby e Cumpata (1986). Esse protocolo apresenta uma escala 
para a codificação da intenção comunicativa da criança, que foi adaptada por Lichtert (2003) e aplicada a crianças surdas. Embora elaborado para a avaliação de linguagem de crianças ouvintes, o autor constatou que o PAPIC tem boa aplicabilidade em crianças surdas de até 30 meses.

Nott et al. (2003) apresentam a técnica chamada Diary of Early Language (Di-EL) que tem o objetivo de obter dados a respeito do desenvolvimento lexical de crianças surdas, com base na investigação de aspectos da oralidade.

Diversos outros pesquisadores têm se empenhado no desenvolvimento de avaliaçōes das línguas de sinais (BERENT, 1990; JANSMA et al., 1997; HERMAN et al., 1999; MALLER et al., 1999; ANDERSON; REILLY, 2002; BEBKO et al., 2003; PEZBINDOWSKI; LEDERBERG, 2003; CAPOVILA; RAPHAEL, 2004; HERMAN et al., 2004; BARBOSA, 2005; LEVY; BARBOSA, 2005; MORGAN, 2006; MORGAN; KEGL, 2006; MORGAN, 2007; QUADROS; CRUZ, 2011), mas ainda há a necessidade de investimentos em pesquisas para o desenvolvimento de instrumentos de avaliação, não apenas no Brasil, mas em diversas partes do mundo (HERMAN et al., 1999; POLLICH, 2001; SCHEMBRI et al., 2002; ABDELFATTAH, 2005; LEVY; BARBOSA, 2005). O impacto de investigações neste campo, dentro da Fonoaudiologia, está diretamente relacionado à sua sofisticação científica e, consequentemente, ao benefício de milhares de pessoas surdas que necessitarão da intervenção do profissional fonoaudiólogo para a otimização dos aspectos relacionados à linguagem.

O protocolo de Avaliação das Habilidades de Comunicação e de Linguagem para Crianças Surdas foi adaptado por Lichtig (1998) para aplicação em crianças surdas brasileiras. Sua aplicação é proposta por Lichtig et al. (2004) como instrumento de avaliação utilizado no Programa de Intervenção Fonoaudiológica em Famílias de Crianças Surdas (PIFFCS). Os estudos realizados focaram crianças surdas na faixa etária de 3 a 6 anos de idade. $O$ protocolo compreende quatro partes: avaliação do perfil pragmático da criança surda (primeira parte), modalidade de comunicação da criança (segunda parte), complexidade linguística (terceira parte) e estilo e eficácia da comunicação na interação entre pai/mãe e criança (quarta parte) (Anexo 1).

Com relação aos objetivos de cada uma das partes do protocolo em questão, temos, na primeira parte, o objetivo de avaliar funções pragmáticas em um ambiente clínico, em interação com o examinador e por entrevista com os pais ou com os responsáveis pela criança. A segunda parte visa analisar a modalidade 
de língua usada preferencialmente pela criança (se é oral, visuoespacial, se usa gestos para se comunicar ou se usa gestos associados a uma dessas modalidades de língua). A terceira parte visa determinar a Média de Extensão das Emissões (Medium Length of Utterances - MLU) e a quarta e última parte tem o objetivo de analisar o estilo comunicativo na interação da criança com seu pai, mãe ou responsável e sua eficácia na transmissão das informações que estes desejam veicular. O procedimento para a aplicação deste protocolo, segundo proposta publicada por Lichtig et al. (2004), será descrito nos métodos.

O objetivo deste estudo é apresentar duas propostas, a saber, uma de redução do protocolo elaborado por Lichtig et al. (2004) e a outra referente a dois tipos de análise dos dados que são obtidos com a aplicação do protocolo reduzido. Essa modificação que será apresentada visa facilitar a análise dos registros da aplicação do protocolo e agilizar sua aplicação, provendo subsídios suficientes para o delineamento de procedimentos terapêuticos e uma medida de evolução terapêutica para fonoaudiólogos, assim como ferramenta para auxiliar na organização de estratégias de intervenção educacional para a criança surda.

\section{Descrição do protocolo original - Protocolo de Avaliação das Habilidades Comunicativas e de Linguagem de Crianças Surdas (LICHTIG et al., 2004)}

O protocolo original elaborado por Lichtig et al. (2004) foi apresentado dentro de um programa chamado de "Programa de Intervenção Fonoaudiológica em Famílias de Crianças Surdas - PIFFCS” (LICHTIG, 2004) no capítulo sobre a avaliação de linguagem de crianças surdas. Para sua apresentação neste trabalho, dividiremos esse protocolo em duas etapas de ação: a Aplicação do protocolo, consistindo nas atividades propostas pelos autores, descritas anteriormente, e os Critérios de Análise, pautados pela folha de registro e análise, dando as diretrizes para interpretar os dados observados no registro (filmagem) obtidos na primeira etapa (Aplicação).

Inicialmente apresentamos a descrição da aplicação e, em seguida, a forma como os dados obtidos devem ser registrados e analisados pelo examinador, de acordo com o que foi proposto pelos autores do protocolo original. 


\section{Parte: Avaliação do Perfil Pragmático da criança surda}

Este item deve ser avaliado em duas situações diferentes: na interação entre o fonoaudiólogo e a criança - situação clínica - e em entrevista com pais ou responsável pela criança. No primeiro momento, realiza-se uma situação de interação semiestruturada para a avaliação do perfil pragmático com as categorias propostas por Dewart e Summers (1994). Na descrição dos procedimentos (LICHTIG et al., 2004), foi proposto o uso de brinquedos em miniaturas como animais e utensílios domésticos, entretanto, sem padronização. São criadas situações de comunicação para observar as modalidades usadas pela criança. Essa situação é registrada através de filmagem. Na segunda situação é efetuada uma entrevista em sala apropriada com os pais ou responsável pela criança, para obtenção de informações sobre a comunicação da criança surda em situações cotidianas. A entrevista também deve ser filmada.

\section{2a. Parte: Modalidade de Comunicação da criança}

São utilizadas como estratégias: (a) a atividade lúdica semiestruturada descrita anteriormente; (b) o uso do vocabulário no momento da interação, com a observação de como a criança requisita os objetos com a interferência mínima do examinador; (c) prova de nomeação, com a apresentação de figuras bidimensionais não padronizadas (avião, casa, vassoura, carro, passarinho, sorvete, peixe, relógio, bola e boneca) quando se pede que a criança diga o que é ou sinalize; (d) história em sequência com quatro cenas, para verificar o tipo de modalidade de comunicação preferencial da criança (oral ou visuoespacial) através da reprodução de uma história contada pelo examinador com base nas cenas já apresentadas na sequência correta (pois não é objetivo verificar a habilidade da criança com relação à noção temporal).

\section{3a. Parte: Complexidade Linguística}

Com a coleta das cinco emissões mais longas produzidas pela criança, é calculada a Média de Extensão das Emissóes (MLU) pela contagem dos vocábulos e aplicação dos critérios descritos pelos autores: vocativos, palavras afirmativas ou negativas, palavras de cumprimento e polidez e repetições são omitidas; morfemas e inflexões são contados separadamente e nomes próprios, palavras compostas, substantivos irregulares, verbos e reduplicaçôes são contados como um elemento. 


\section{4a. Parte: Estilo e eficácia da comunicação na interação entre pai/mãe e criança}

Avaliação de situação de interação entre a criança e a mãe (ou pai ou responsável), com a solicitação de seis tarefas usando blocos de diferentes tamanhos e cores. Sugere-se o registro filmado da interação para posterior análise. O examinador orienta o interlocutor da criança nas solicitaçôes que apresentará.

Para a análise dos dados obtidos, sugere-se o registro na Folha de Registro e Análise proposta pelos autores. Em relação ao perfil pragmático, os eventos devem ser classificados de acordo com as habilidades comunicativas propostas por Dewart e Summers (1994) e de acordo com o tipo de modalidade em que ocorreu (língua oral, língua de sinais, gesto, vocalização). A análise da segunda parte (Modalidade de Comunicação da Criança) é feita através de quatro tópicos descritos anteriormente e que devem ser registrados e classificados de acordo com as modalidades comunicativas.

A terceira parte (Complexidade Linguística), obtida através do Medium Length of Utterances (MLU) é realizada com a média de morfemas das maiores sentenças produzidas pela criança de acordo com critérios estabelecidos para o Português Brasileiro. Não há sugestão de critérios para cálculo do MLU em língua de sinais.

A quarta parte (Estilo e Eficácia da Comunicação na Interação entre Pai/ Mãe e Criança) baseia-se na análise da interação entre a criança e seu interlocutor verificando a eficácia dessa comunicação na transmissão de ordens à criança. Não há pontuação sugerida, apenas a marcação no quadro proposto.

Os autores também sugerem a transcrição integral dos registros da aplicação do protocolo. O protocolo tem o caráter de cheklist, e não é objetivo delinear diagnóstico de linguagem, mas o perfil comunicativo e de linguagem da criança surda.

\section{Resultados e discussão}

As sugestôes de alterações do Protocolo do PIFFCS estão apresentadas em seguida, assim como a discussão dessas alteraçôes. É importante enfatizar que esta proposta não exclui nem desmerece a aplicação do protocolo original, apenas apresenta uma forma diferente e complementar para a avaliação da comunicação e linguagem de crianças surdas. Por esse motivo, denominamos o protocolo proposto "Protocolo de Avaliação das Habilidades Comunicativas e de Linguagem de Crianças Surdas Reduzido" (Protocolo do PIFFCS-reduzido). 


\section{Apresentação da proposta do Protocolo de Avaliação das Habilidades Comunicativas e de Linguagem de Crianças Surdas Reduzido (Protocolo do PIFFCS-reduzido)}

O protocolo que propomos neste trabalho está baseado no protocolo elaborado por Lichtig et al. (2004), chamado de "Avaliação das Habilidades Comunicativas e de Linguagem de Criança Surdas" (Protocolo do PIFFCS). Embora a proposta que apresentamos possa ser considerada uma modificação do protocolo PIFFCS, a utilização deste continua sendo válida para a avaliação de comunicação e linguagem em crianças surdas. Consideramos que a apresentação deste novo protocolo consiste em uma redução daquele em que se baseou, tendo como objetivo uma aplicação mais rápida e que pode ser realizada por profissionais com e sem o domínio da Língua de Sinais Brasileira.

O protocolo que apresentamos neste trabalho, detalhando as modificações que foram feitas comparativamente ao protocolo original, será denominado Protocolo do PIFFCS - reduzido, enfatizando que esta proposta não é uma criação de um novo protocolo, mas o desdobramento reduzido daquele elaborado por Lichtig et al. (2004). Em nossa proposta, dividimos o Protocolo do PIFFCS - reduzido em três partes: $1^{\mathrm{a}}$. Parte (Interação Entre Examinador e Criança), $2^{\mathrm{a}}$. Parte (Modalidade de Comunicação com Estímulos Visuais Específicos) e $3^{\text {a }}$. Parte (Complexidade Linguística). Para a análise destas três partes que compõem o protocolo, sugerimos a observação ou o registro (em vídeo) de dois momentos, que seriam o momento de interação entre o examinador e a criança e a apresentação de estímulos visuais específicos (figuras e história em sequência), da mesma forma como foi proposto por Lichtig et al. (2004).

As sugestóes de alteração serão propostas tanto para a Aplicação quanto para os Critérios de Análise do Protocolo do PIFFICS (original). Para a etapa de Aplicação, as alterações se concentraram na segunda e terceira partes do protocolo original proposto por Lichtig et al. (2004). Na segunda parte (Modalidade de Comunicação da Criança), é sugerida a tarefa de nomeação de dez figuras específicas e uma história em sequência com quatro figuras. Sugerimos alteração para trinta figuras, contemplando campos semânticos diferentes, selecionadas do Teste de Vocabulário do ABFW (BEFI-LOPES, 2000) e uma história em seqüência com cinco figuras. Estas sugestões são feitas com o objetivo de aumentar o número de observações de produção da criança sob um estímulo visual específico (nesse caso, a figura a ser nomeada ou sinalizada), contemplar os campos semânticos propostos por um teste 
de vocabulário padronizado e aumentar o número de eventos expostos nas figuras da sequência. Na terceira parte (Complexidade Linguística), em que os autores do protocolo original sugerem a análise do MLU, propomos a utilização das fases linguísticas descritas por Quadros (1997). Esta alteração foi realizada para diminuir o tempo necessário para a análise dos dados coletados na avaliação. Nas demais partes não houve alterações.

$\mathrm{Na}$ aplicação da história em sequência, o enunciado do pedido para a recontagem foi alterado. No procedimento sugerido por Lichtig et al. (2004) para que a criança conte a história, o examinador conta a história exibida na sequência e apresenta as figuras na sequência correta. Propomos que o examinador apenas peça à criança que organize as figuras em sequência e que conte o que acontece nelas. Esse procedimento facilita a aplicação para examinadores que não dominam a língua de sinais e diminui a possibilidade de a criança copiar a produção realizada pelo seu examinador.

Na prova de nomeação foram utilizadas 30 figuras selecionadas do teste de Vocabulário do ABFW (BEFI-LOPES, 2000), contemplando todos os campos semânticos. A seleção das figuras dentre as sugeridas pelo autor do Teste de Vocabulário foi realizada por sorteio, de forma proporcional entre os campos semânticos contemplados pelo teste.

$\mathrm{Na}$ "Folha de Registro e Análise" proposta por Lichtig et al. (2004) para a organização das informações coletadas na aplicação e sua análise, propomos nova formatação e reformulação que podem facilitar o registro dos dados e organizar de forma mais clara suas análises.

No Protocolo do PIFFCS, a análise da primeira parte da avaliação (Perfil Pragmático), segundo a proposta original, utiliza as categorias propostas por Dewart e Summers (1994) e deve-se anotar no quadro proposto (Anexo, $1^{2}$ Parte) se houve a ocorrência em gesto, língua oral, língua de sinais, vocalização ou se não ocorreu aquela categoria no momento de interação filmado.

Propomos, inicialmente, a modificação da disposição do quadro com relação à divisão das categorias e sua tabulação com as modalidades de comunicação previstas para a ocorrência (gesto, língua de sinais, vocalização, língua oral). As categorias pessoa, lugar, tempo, assunto, uso da linguagem, interação, livros e regras sociais são observadas mais facilmente em contextos extraclínicos e sua especificação de uso deve ser realizada de forma qualitativa.

A marcação das ocorrências em gesto, língua de sinais, vocalização e língua oral em um quadro simples podem não computar a ocorrência de mais de uma 
modalidade em um mesmo evento (modalidade associada, bimodalismo), e deixar dúbia a ocorrência de uma modalidade isolada ou ao mesmo tempo que as outras. Por exemplo, se uma criança em um dado momento da interação exibe a habilidade comunicativa "comentando" em gesto e logo em seguida exibe a mesma habilidade comunicativa em gesto e vocalização, a marcação no quadro proposto pelo protocolo original não deixa espaço suficiente para separar o momento em que a criança realizou uma modalidade única (o gesto) e a bimodalidade (o gesto mais a vocalização).

Propomos, então, a retirada das habilidades comunicativas de Variação do Contexto (itens 26 a 33, observados no primeiro quadro do Anexo 1) e que as modalidades observadas se agrupem não mais em Vocal, Gesto, Língua de Sinais e Língua Oral, mas em Modalidade Visuoespacial (MVE), Modalidade Oral Auditiva (MOA) e Bimodalidade (BM), o que permitirá a maior precisão com relação à ocorrência das modalidades (ver Anexo 2).

Para a entrevista proposta no protocolo original, a Variação Contextual pode ser investigada, entretanto, de forma qualitativa e sem a obrigatoriedade de marcação de modalidade, já que contempla, em alguns tópicos, outros tipos de resposta, como em "livros" e "pessoa". Esta parte do protocolo foi retirada.

As modificaçōes foram propostas com o objetivo de facilitar o registro e análise dos dados, tornando-os rápidos e acessíveis aos fonoaudiólogos com conhecimentos básicos na Língua de Sinais Brasileira (LSB).

A marcação da pontuação no quadro deve acontecer no momento em que o examinador observa o registro da coleta, registrando apenas a ocorrência ou não de uma determinada habilidade comunicativa, sendo que esse registro também fornecerá a informação sobre qual foi a modalidade usada naquela ocorrência (o que seria um checklist com uma informação adicional).

O registro da ocorrência das habilidades comunicativas nas modalidades, então, deve ser marcado com a observação do registro filmado, assinalando no local adequado quais são as habilidades comunicativas apresentadas pela criança e em que modalidade essa habilidade comunicativa se manifestou, não sendo computada a frequência da ocorrência, mas apenas a ocorrência ou não delas.

Para a marcação da pontuação do desempenho dos sujeitos, propomos dois tipos de análise: Tipo I e Tipo II. Essa diferenciação do tipo de pontuação utilizada deve estar relacionada ao grau de domínio da língua de sinais pelo examinador.

Análise do Tipo I - pode ser realizada por profissionais sem domínio da língua de sinais, que possuem conhecimentos rudimentares, mas que diferenciem os tipos 
de modalidade (Modalidade Visuoespacial, Modalidade Oral-Auditiva e Bimodalidade) na produção da criança surda. Marca-se apenas a ocorrência ou não do evento, sem quantificar sua frequência ou qualificar a complexidade da comunicação da criança. Nesse tipo de análise há a possibilidade de ocorrerem dois perfis parecidos (por exemplo, para as ocorrências em MVE), mas com complexidades diferentes (um usa gesto e outro língua de sinais), mas é uma aplicação que pode dar, de forma segura, o perfil da modalidade predominante na comunicação da criança, mesmo que o profissional não seja fluente em língua de sinais.

Análise Tipo II - deve ser aplicada pelo profissional com domínio na língua de sinais. A marcação também é a ocorrência da modalidade para o estímulo ou para o tipo de habilidade comunicativa que ocorre, mas com uma pontuação específica que qualifica a resposta em sua complexidade linguística. Marca-se, então, um ponto (1) para respostas em que não se usa língua (gesto em MVE, vocalização em MOA e gesto com vocalização em $\mathrm{BM}$ ) e dois pontos (2) para respostas em que ocorre uso de uma língua (língua de sinais em MVE, língua oral em MOA e uma ou duas destas em BM). No Perfil Pragmático, em que há a marcação do tipo de modalidade dentro da ocorrência de uma habilidade comunicativa, marca-se apenas uma ocorrência para cada habilidade comunicativa em cada modalidade, no caso de ocorrer mais de uma modalidade por habilidade comunicativa. Para qualificar a complexidade da modalidade ocorrida, predomina a marcação de maior complexidade (língua), quando esta ocorrer pelo menos uma vez em toda a produção da criança. Por exemplo, uma criança que apresentou a ocorrência nomeando em MVE no início da interação com gesto, marca-se 1 na célula apropriada; ocorrendo em seguida o uso de língua de sinais para a mesma habilidade, troca-se a marcação de 1 para 2, pois houve o uso de língua em MVE. Isso deve ocorrer em todas as marcações. Para a pontuação em Nomeação, o procedimento deve ser repetido, entretanto observando a resposta específica para cada estímulo apresentado, não sendo considerados os processos semânticos de vocabulário e as não desinências (quando a criança não atribui sinal ou nome), mas apenas a modalidade comunicativa usada dentro das respostas obtidas.

$\mathrm{Na}$ análise Tipo I, a porcentagem deve ser obtida pela compatibilização do valor máximo de cada coluna, obtendo-se as pontuações percentuais que delinearão o perfil de uso de modalidade e o perfil pragmático pela ocorrência ou não das Habilidades Comunicativas que também podem ser analisadas de acordo com os grupos de categorias, a saber, Intençōes Comunicativas, Respostas à Comunicação e Interação e Conversação. 
A análise do Tipo II deve ser feita com relação às médias de pontuação. Esse tipo de análise pode sugerir duas informaçôes a respeito da criança avaliada: (1) o nível de desenvolvimento em que a criança se encontra em sua aquisição de língua, ou seja, se ela tende a usar mais língua, ou código estruturado, aproximando-se da pontuação 2, ou se ela tende a usar mais gestos ou vocalizações, aproximando-se da pontuação 1; e (2) o desenvolvimento da criança após período em terapia, comparando-se a média obtida antes e depois de um período de intervenção fonoaudiológica.

Com o objetivo de facilitar a análise do protocolo por fonoaudiólogos não fluentes na LSB, sugerimos, em conformidade com o caráter de checklist do instrumento, a observação da produção da criança e a marcação nos campos adequados e referentes às modalidades usadas, em um quadro elaborado para este estudo, que pode ser observado no Anexo, quadro 1.2.

A Complexidade Linguística é apresentada pelos autores do protocolo, com a análise da MLU da produção da criança. Esse tipo de análise exige domínio pleno da língua a ser analisada, portanto a análise da produção em PB seria possível, mas acarretaria gasto de tempo para transcrição total da produção da criança nas provas. Propomos, então, que a análise da Complexidade Linguística seja realizada através da observação da produção da criança em todo o desenvolvimento da aplicação do protocolo e a marcação dessa análise seja feita com base nas fases propostas por Quadros (1997) com registro no quadro de marcação específico (Anexo, 3a. Parte). Essas fases são: Período Pré Linguístico, Estágio de um Sinal, Estágio das Primeiras Combinações e Estágio das Múltiplas Combinações.

Segundo Quadros (1997), o Período Pré-Linguístico inicia-se no nascimento e decorre até a aquisição do primeiro sinal, com diferenças de transição para o período linguístico não tão claras (WOLL, 1998). O marco de distinção entre o período pré-linguístico e o período linguístico fica relacionado à produção do primeiro sinal articulado no padrão de sinalização adulto (QUADROS, 1997). O Estágio de um Sinal inicia-se por volta dos 12 meses e dura até aproximadamente 24 meses (QUADROS, 1997). São considerados para a identificação do início do estágio os dez primeiros sinais. Nesse estágio, há intenso desenvolvimento de vocabulário; a aquisição dos sinais ocorre em número similar ao apresentado pelas crianças ouvintes na aquisição de palavras, de acordo com a faixa etária (WOLL, 1998; QUADROS, 1997). Ainda nesse estágio, o "apontar" muda seu uso, tomando um caráter linguístico. O Estágio das Primeiras Combinações é aquele que ocorre por volta dos dois anos de vida da criança quando surgem as primeiras 
combinações de sinais. Inicia-se a utilização de sistema pronominal de forma inconsistente. Na LSB observa-se o uso de combinações de dois a três sinais, omissóes de sujeito, uso de formas verbais que pedem concordância de forma inadequada e uso adequado de pronomes estabelecidos no espaço de sinalização. No Estágio das Múltiplas Combinações, segundo Quadros (1997), que ocorre por volta dos dois anos e meio, as crianças surdas apresentam a "explosão de vocabulário" e começam a distinguir formas derivadas, como "sentar" e "cadeira" (que correspondem a um mesmo sinal). O domínio completo dos recursos morfológicos ocorre por volta dos cinco anos de idade. Com três anos de idade, as crianças começam a usar o sistema pronominal para referência a entidades não presentes no contexto, porém apresentam erros.

Esses estágios foram baseados em estudos realizados com crianças surdas, entretanto, devido ao seu caráter genérico, podem ser aplicados ao desenvolvimento da língua oral (QUADROS, 1997).

A entrevista realizada com o responsável pela criança e a interação entre esse responsável e a criança podem ser analisadas seguindo os mesmos critérios propostos no Protocolo do PIFFCS. No protocolo PIFFCS-reduzido, essas etapas da avaliação foram retiradas, por isso, para a obtenção de dados extraclínicos, deve-se recorrer ao PIFFCS original.

Com essas alterações, a Folha de Registro e Análise ganha uma outra formatação, que segue exemplificada no Anexo 2 deste trabalho.

\section{Considerações finais}

Na realização de avaliação de linguagem de crianças surdas, o cuidado na observância das habilidades desenvolvidas com base na língua de sinais não pode ser uma opção profissional a depender da visão sobre a surdez, por exemplo, com relação a que tipo de estimulação linguística (oral ou de sinais) deve ser oferecida como base para aquisição da primeira língua. Mesmo atualmente, com o desenvolvimento acelerado de recursos tecnológicos para a reabilitação auditiva - a visão que pretende normalizar a atividade linguística de uma criança que nasce surda -, a língua de sinais tem se mostrado relevante no desenvolvimento das habilidades de linguagem de crianças surdas que usam língua de sinais ou que usam uma língua oral.

Quando não se conhece o perfil de linguagem da criança, o risco da escolha de procedimentos inadequados é grande e as consequências da aplicação desses 
procedimentos podem impactar o seu desenvolvimento humano, extrapolando o domínio linguístico e atenuando, por exemplo, seu desenvolvimento acadêmico, social e profissional. Com um instrumento que permite a visualização das habilidades linguísticas baseadas na aquisição da língua de sinais, o profissional que lida com a criança surda tem mais uma ferramenta para compreender as necessidades e habilidades reais da criança no que diz respeito à linguagem. Compreendendo esse perfil, os achados podem ser usados para o benefício da criança no desenvolvimento terapêutico ou na prática educativa, potencializando suas habilidades no uso da língua, impedindo que restrições no acesso à informação ocorram e provendo base para seu desenvolvimento humano pleno.

\section{Referências}

ABDEL-FATTAH, M.A. Arabic Sign Language: a perspective. Journal for Deaf Studies and Deaf Education, n. 10, v. 2, p. 212-21, 2005.

ALPINER, J.G.; MACCARTHY, P.A. Rehabilitative audiology: childrens and adults. Baltimore: Williams e Williams, 1987.

ANDERSON, D.; REILLY, J. The MacArthur Communicative Development Inventory: normative data for american sign language. Journal of Deaf Studies and Deaf Education, n. 72, p. 83-119, 2002.

ATKINSON, J.; MARSHALL, J.; WOLL, B.; TRACKER, A. Testing comprehension abilities in users of British Sign Language following CVA. Brain Lang, n. 94, v. 2, p. 233-48, 2005.

BALBANI, A.P.S.; MANTOVANI, J.C. Impacto das otites médias na aquisição de linguagem. Jornal de Pediatria. n. 79, v. 5, p. 391-96, 2003.

BARBOSA, F.V. Refletindo sobre o novo. Rev Soc Bras Fonoaudiol, 2005. [Comentário de: Marshall J, Atkinson JR, Woll B, Tracker A. Aphasia in a bilingual user of British Sign Language and English: effect of cross linguistic cues. J. Cogn Neuropsychology, n. 22, v. 6, p. 719-36, 2005.]

BARBOSA, F. V.; NAVAS, A. L. G. P.; ENOMOTO, C. M.; PANSONATO, R. D. L; SANTOS, Z. M. Propostas para a avaliação do sistema fonológico da Língua de Sinais Brasileira: estudos iniciais (resumo). Anais do XIII Congresso Brasileiro de Fonoaudiologia. Sociedade Brasileira de Fonoaudiologia. Santos, 2005. Barbosa, 2006.

BALBANI, A. P. S.; MANTOVANI, J. C. Impacto das otites médias na aquisição de linguagem. Jornal de Pediatria. 2003. 79(5). 
BEBKO, J. M.; CALDERON, R.; TREDER, R. The Language Proficiency Profile -2 : assessment of the global communications skills of deaf children across languages and modalities of expression, n. 8, v. 4, p. 438-51, 2003.

BEFI-LOPES, D. Avaliação de vocabulário. In: ANDRADE, C.R.F.; BEFI-LOPES, D.M.; FERNANDES, F.D.M.; WERTZNER, H.F. $A B F W$ - Teste linguagem infantil nas ares de fonologia, vocabulário, fluência e pragmática. Barueri: Pró-Fono; 2000. p.33-49.

BERENT, G.P. An Assessment of syntatic capabilities. In: M. STRONG. Language learning and deafness. Cambridge: Cambridge University Press, 1990.

BORGES, L.C.; SALOMÃO, N.M.R.. Aquisição de linguagem: considerações da perspectiva da interação social. Psicologia: Reflexão e Crítica, n. 16, v. 2, p. 327-36, 2003. BRAZ, H. A.; PELLICCIOTTI, T. H. F. Exame de Linguagem TIPITI. São Paulo: MNJ, 1981.

CAPOVILA, F. C.; RAPHAEL, W. D. Enciclopédia da Lingua de Sinais Brasileira - o mundo surdo em libras. Sinais da Libras e o universo da educação, como avaliar o desenvolvimento da capacidade de leitura de palavras (processos de reconhecimento e decodificação) em escolares surdos do ensino fundamental ao médio. São Paulo: EDUSP, 2004. 1v.

CARVALHO, A. Efeito do acender e do apagar de pistas periféricas sobre o tempo de reação manual para alvos visuais em ouvintes e surdos. 2005. Dissertação (Mestrado) - Universidade Federal Fluminense, Niterói, 2005.

CASBY, M.W.; CUMPATA, J.F. A protocol for the assessment of prelinguistic intentional communication. J Commun Disord, n. 19, p. 251-60, 1986.

DE RENZI, E.; VIGNOLO, L.A. The Token Test: a sensitive test to detect receptive disturbances in aphasics. Brain, n. 84, p. 665-78, 1962.

DEWART, H.; SUMMERS, S. The pragmatics profile of early communication skills. 2. ed. Windsor: NFER-Nelson, 1994.

FERNANDES, F.D.M. Avaliação pragmática. In: ANDRADE, C.R.F.; BEFI-LOPES, D.M.; FERNANDES, F.D.M.; WERTZNER, H.F. $A B F W$ - Teste linguagem infantil nas áreas de fonologia, vocabulário, fluência e pragmática. Barueri: Pró-Fono, 2000. p. 83-97. FERREIRA-BRITO, L. Por uma gramática da Lingua de Sinais. Rio de Janeiro: Editora Tempo Brasileiro, 1995.

GRIMSHAW, G.M.; ADELSTEIN, A.; BRIDEN, M.P.; MACKINNON, G.E. First language acquisition in adolescence: evidence for a critical period of verbal language development. Brain Lang, n. 63, p. 237-55, 1998. 
HERMAN, R. The need for an assessment of deaf children's signing skills. Deafness and Education, n. 22, p. 3-8, 1998.

HERMAN, R.; HOLMES, S.; WOLL, B. Assessing BSL development - receptive skills test. Coleford: The Forest Bookshop, 1999.

JANSMA, S.; KNOORS, H.; BAKER, A.E. Sign Language assessment: a dutch project. Deafness and Education. Journal of the British Association of the Teachers of the Deaf, n. 21, v. 3, p. 39-46, 1997.

KAPLAN, E.; GOODGLASS, H.; WEINTRAUB, S. Teste de Boston para diagnóstico de la afasia. Adaptação española de García-Albea e Sánchez Bernardos. Madri: Editora Panamericana, 1996.

LEVY, C.C.A.C.; BARBOSA, F.V. Surdez: condutas na avaliação e planejamento terapêutico. In: LOPES-FILHO, O. Tratado de Fonoaudiologia. São Paulo: TecMed, 2005. p.377-89.

LICHTERT, G. Assessing intentional communication in deaf toddlers. Journal for Deaf Studies and Deaf Education, n. 8, v. 1, p. 43-56, 2003.

LICHTIG, I. Propostas alternativas de identificação e intervenção fonoaudiológica com crianças deficientes auditivas. Tese (Livre-docência). Faculdade de Medicina, Universidade de São Paulo, São Paulo, 1998.

LICHTIG, I. (Org.). Programa de intervenção fonoaudiológica com famílias de crianças surdas (PIFFCS). Carapicuíba: Pró-Fono, 2004.

LICHTIG, I.; COUTO, M.I.V.; MECCA, F.F.D.N. Avaliando as habilidades comunicativas e lingüísticas de crianças surdas. In: LICHTIG I. (Org.). Programa de intervenção fonoaudiológica com familias de crianças surdas (PIFFCS). Carapicuíba: Pró-Fono; 2004. p. 39-78.

LODI, A.C.B. Educação bilíngüe para surdos. In: LACERDA, C.B.F.; NAKAMURA, H.; LIMA, M.C. Fonoaudiologia: surdez e abordagem bilingüe. São Paulo: Plexus, 2000. p. 64-83.

MALLER, S.J.; SINGLETON, J.L.; SUPALLA, S.J.; WIX, T. The development and psychometric properties of the American Sign Language-Proficiency Assessment (ASL-PA). Journal of Deaf Studies and Deaf Education, n. 4, v. 4, p. 249-69, 1999.

MOGFORD, E.; BISHOP, D. O Desenvolvimento da Linguagem em Circunstâncias Excepcionais. Rio de Janeiro: Revinter, 2002.

MORGAN, G.; KEGL, J. Nicaraguan sign language and theory of mind: the issue of critical periods and abilities. Journal of Child Psychology and Psychiatry, n. 47, p. 811-19, 2006. 
MORGAN, G. Children are just lingual: The development of phonology in BSL. Lingua, n. 116, p. 1507-23, 2006.

MORGAN, G. The first signs of language: phonological development in British Sign Language. Applied Psycholinguistics, n. 28, v. 3, p. 22, 2007.

NICHOLAS, J.G.; GEERS, A.E. Hearing status, language modality and youg children's comunicative and linguistic behaviour. Journal for Deaf Studies and Deaf Education, n. 8, p. 422-37, 2003.

NOTT, P.; COWAN, R.; BROWN, P.M.; WIGGLESWORTH, G. Assessment of language skills of young children with profound hearing loss under two years of age. Journal for Deaf Studies and Deaf Education, n. 8, v. 4, p. 401-21, 2003.

PEZBINDOWSKI, A.K.; LEDERBERG, A. R. Vocabulary assessment of deaf and hard-of-hearing children from infancy through the preschools years. Journal for Deaf Studies and Deaf Education, n. 8, v. 4, p. 384-400, 2003.

PICKERSGILL, M. Bilingualism, current policy and practice. In: GREGORY, S.; KNIGHT, P.; MCCRACKEN, W.; POWERS, S.; WATSON, L. Issues in deaf education. London: David Fulton Publishers, 1998. p. 88-97.

POLLICH, L. Education of the Deaf in Nicarágua. Journal for Deaf Studies and Deaf Education, n. 6, v. 4, p. 315-26, 2001.

QUADROS, R. M. Educação de surdos: aquisição da linguagem. Porto Alegre: Artes Médicas, 1997.

QUADROS, R. M.; CRUZ, C. R. Lingua de Sinais: Instrumentos de Avaliação. Porto Alegre: Editora ArtMed, 2011.

QUADROS, R. M.; KARNOPP, L. B. Lingua de sinais brasileira-estudos lingüisticos. Porto Alegre: ArtMed, 2004.

SCHEMBRI, A. et al. Issues in development of the test battery for australian sign language morphology and sintax. Journal for Deaf Studies and Deaf Education, n. 7, v. 1, p. 19-40, 2002.

WOLL , B. Development of signed and spoken languages. In: GREGORY, S; KNIGHT, P.; McCRACKEN, W.; POWERS, S.; WATSON, L. Issues in deaf education. London: David Fulton Publishers, 1998. p. 58-68. 


\section{ANEXO 1}

\begin{tabular}{|c|c|c|c|c|c|c|c|c|c|c|}
\hline \multirow{2}{*}{\multicolumn{11}{|c|}{$\begin{array}{c}\text { do PIFFCS ( 2004) } \\
\text { Foltha de Registro e Analise }\end{array}$}} \\
\hline & & & & & & & & & & \\
\hline \multicolumn{11}{|c|}{ 12. Parte: PERFIL PRAGMLitICO } \\
\hline TOPICOS & \multicolumn{5}{|c|}{ OBSERVAÇAOO } & \multicolumn{5}{|c|}{ ENTREVISTA } \\
\hline & Gesto & Voc. & LO & LS & N.A. & Gesto & Voc. & LO & LS & N.A. \\
\hline 1. Atençio & & & & & & & & & & \\
\hline 2. solucitando & & & & & & & & & & \\
\hline 3. rejeitando & & & & & & & & & & \\
\hline 4.cumprimentando & & & & & & & & & & \\
\hline 5. expressando & & & & & & & & & & \\
\hline 6. nomeando & & & & & & & & & & \\
\hline 7. comentando & & & & & & & & & & \\
\hline 8. informando & & & & & & & & & & \\
\hline 9. obtendo inform. & & & & & & & & & & \\
\hline 10. mteresse & & & & & & & & & & \\
\hline 11. compr. gestes & & & & & & & & & & \\
\hline 12. reconhecendo & & & & & & & & & & \\
\hline 13. comp intengles & & & & & & & & & & \\
\hline 14. antecipachio & & & & & & & & & & \\
\hline 15. respondendo & & & & & & & & & & \\
\hline 16. aegociando & & & & & & & & & & \\
\hline 17. iniciando & & & & & & & & & & \\
\hline 18. manteado & & & & & & & & & & \\
\hline 19. inteligibilidade & & & & & & & & & & \\
\hline 20. pressuposiç̧o & & & & & & & & & & \\
\hline 21. гераяо & & & & & & & & & & \\
\hline 22. pedido claseza & & & & & & & & & & \\
\hline 23. terminando & & & & & & & & & & \\
\hline $24 . c a v i n d 0$ & & & & & & & & & & \\
\hline 25. participando & & & & & & & & & & \\
\hline 26. pessoa & & & & & & & & & & \\
\hline 27. lugex & & & & & & & & & & \\
\hline 28. tempo & & & & & & & & & & \\
\hline 29. assuanto & & & & & & & & & & \\
\hline 30.uso linguagem & & & & & & & & & & \\
\hline 31. interacio & & & & & & & & & & \\
\hline 32. Lnxos & & & & & & & & & & \\
\hline 33. regras sociais & & & & & & & & & & \\
\hline
\end{tabular}

Legenda: Voc: vocalizaç̧o / LO: Lingua Oral / LS: Liagua de Sinass / N.A: Nåo Apresentou

FIGURA 1 
$2^{a}$. Parte: MODALIDADE DE COMUNICAÇÃO E FASE LINGUISTIICA

2.1. Uso do Vocabulário

2.2. Nomeação

\begin{tabular}{|l|l|l|}
\hline Figuras & Emissão & Observação (modalidade) \\
\hline 1. sorvete & & \\
\hline 2. relógio & & \\
\hline 3. casa & & \\
\hline 4. avião & & \\
\hline 5. carro & & \\
\hline 6. bola & & \\
\hline 7. boneca & & \\
\hline 8. peixe & & \\
\hline 9. vassoura & & \\
\hline 10. passarinho & & \\
\hline
\end{tabular}

3. Estória em Sequência

Transcrição:

\begin{tabular}{|l|l|l|l|l|l|}
\hline Gestos & Vocalização & Gestos/voc. & Língua Oral & Língua de Sinais & Lingua Oral e Lingua de Sinais \\
\hline & & & & & \\
\hline
\end{tabular}

a. Parte: COMPIEXIDADE LINGUISTICA

3.1. Exemplos das emissões mais longas ( minimo 3): $\quad 3.2 . \mathrm{MLU}=$

$4^{\text {a }}$. Parte: ESTILO E EFICÁCIA DA COMUNICAÇÃO

4.1. Eficácia na transmissão das tarefas

\begin{tabular}{|l|l|l|l|}
\hline Tarefa & & Realizou & Não Realizou \\
\hline 1 & Dá un bloco pequeno & & \\
\hline 2 & Dá unn bloco azul & & \\
\hline 3 & Põe o bloco pequeno emb aixo do grande & & \\
\hline 4 & Põe o bloco amarelo em cima do azul & & \\
\hline 5 & Põe o bloco pequeno e amarelo ao lado do bloco pequeno azul & & \\
\hline 6 & Põe o bloco grande e vermelho em cina da cadeira e dá o bloco azul & & \\
\hline
\end{tabular}

4.2. Estilo de comunicação

\begin{tabular}{|c|c|c|c|c|}
\hline \multirow{2}{*}{$\begin{array}{l}\text { Estratégias de comunicação } \\
\text { A mãe obtém a atenção da criança antes de iniciar a } \\
\text { comunicação? }\end{array}$} & Sempre & \begin{tabular}{|l|} 
Freqüentemente \\
\end{tabular} & Raramente & Nunca \\
\hline & & & & \\
\hline \multicolumn{5}{|l|}{ A mãe sinaliza, fala e aponta ao mesmo tempo? } \\
\hline \multicolumn{5}{|l|}{ A criança atende a mãe ou faz troca de turno? } \\
\hline $\begin{array}{l}\text { A mãe obtém a atenção da criança através de: } \\
\text { a) contato fisico leve? } \\
\text { b) contato fisico brusco? } \\
\text { c) Toque, sinais ou gestos em seu campo visual? } \\
\text { d) Utiliza o acerro? }\end{array}$ & & & & \\
\hline \multicolumn{5}{|l|}{$\begin{array}{l}\text { A criança recusa ou desiste de fazer uma ou todas as } \\
\text { tarefas? }\end{array}$} \\
\hline $\begin{array}{l}\text { A mãe fala para a criança o que deve ser feito antes de } \\
\text { iniciar a tarefa? }\end{array}$ & & & & \\
\hline
\end{tabular}

Resultado:

COMENTÁRIOS:

CONCLUSÕES:

FIGURA 2 


\section{ANEXO 2}

Protocolo de Avaliação das Habilidades Comunicativas e de Linguagem de Crianças Surdas Reduzido (Protocolo do PIFFCS - reduzido)

Nome: D.N.

Folha de Registro e Análise - Aplicação do protocolo realizado em: ( ) LS ( ) PB ( ) BM ( ) G

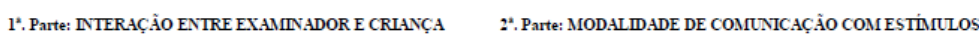

\begin{tabular}{|c|c|c|c|c|}
\hline TOPICOS & \multicolumn{4}{|c|}{ 1.1. Perfil Pragmático } \\
\hline \multicolumn{5}{|c|}{ 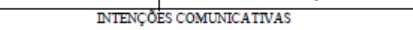 } \\
\hline & MVE & MOA & BM & $\mathrm{NA}$ \\
\hline \multirow{2}{*}{\multicolumn{5}{|c|}{ 1. atenç̧⿰丿𠃌 }} \\
\hline 2. solicitando & & & & \\
\hline \multicolumn{5}{|l|}{ 3. rejeitzndo } \\
\hline \multicolumn{5}{|l|}{ 4.cumprimentan do } \\
\hline \multicolumn{5}{|l|}{ 5. expressando } \\
\hline \multicolumn{5}{|l|}{ 6. nomendado } \\
\hline \multirow{2}{*}{\multicolumn{5}{|c|}{ 7. comentando }} \\
\hline \multicolumn{3}{|l|}{8 informando } & & \\
\hline \multirow{2}{*}{\multicolumn{5}{|c|}{\begin{tabular}{l|l|l|l} 
Subtotal & & & \\
RESPOSTAS PARA A COMCNICAÇAO
\end{tabular}}} \\
\hline & & & & \\
\hline \multicolumn{5}{|c|}{ 9.obtendo inform. } \\
\hline \multicolumn{5}{|l|}{ 10. interesse } \\
\hline \multicolumn{5}{|l|}{ 11. compr. Gestos } \\
\hline \multicolumn{5}{|l|}{12 reconherendo } \\
\hline \multicolumn{5}{|l|}{13 .comp intençóes } \\
\hline \multicolumn{5}{|l|}{ 14. antecipuşio } \\
\hline \multirow{2}{*}{\multicolumn{5}{|c|}{ 15. respondendo }} \\
\hline \multicolumn{3}{|l|}{ 16. negociundo } & & \\
\hline \multirow{2}{*}{\multicolumn{5}{|c|}{ INIERAÇAO E CONVERSAÇAOAO }} \\
\hline & & & & \\
\hline \multicolumn{5}{|l|}{ 17. iniciendo } \\
\hline \multicolumn{5}{|l|}{ 18. mantendo } \\
\hline \multicolumn{5}{|l|}{ 19. inteligibilidadade } \\
\hline \multicolumn{5}{|l|}{ 20. pressuposiç̧o } \\
\hline \multicolumn{5}{|l|}{ 21. reparo } \\
\hline \multicolumn{5}{|l|}{22 pedido clareza } \\
\hline \multicolumn{5}{|l|}{ 23. terminando } \\
\hline \multicolumn{5}{|l|}{ 24. ouvindo } \\
\hline \multicolumn{5}{|l|}{ 25. participindo } \\
\hline Subotal & & & & \\
\hline Total & & & & \\
\hline
\end{tabular}

\begin{tabular}{|c|c|}
\hline \multicolumn{2}{|c|}{ 1.2. Ocorrência de Código Estruturado } \\
\hline \multirow{2}{*}{ MNE } & Lingua de Sinnis \\
\hline & Gestos \\
\hline \multirow[t]{2}{*}{ MOA } & Lingua Oral \\
\hline & Vocalização \\
\hline \multirow[t]{4}{*}{ BM } & Lingua de Sinais \\
\hline & Gestos \\
\hline & Lingua Oral \\
\hline & Vocalização \\
\hline
\end{tabular}

MVE: Modalidade Viso Espacial; MOA: Modalidade Oral Auditiva; BN Bimodalidade; LO: Lingua Ora; LS: Língua de Sinzais; G. Gestos; NA: Não

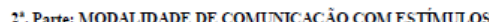
VTSUAIS ESPECIFICOS

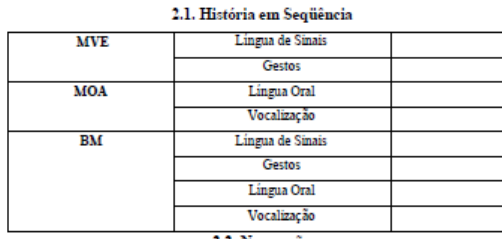
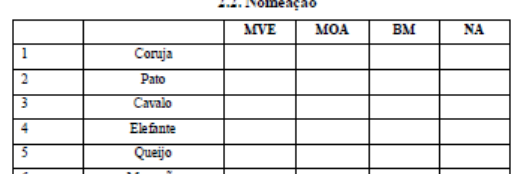

\begin{tabular}{|l|c|l|l|l|l|}
\hline 7 & Bunana & & & & \\
\hline 8 & Abucasi & & & & \\
\hline 9 & Helicoptero & & & & \\
\hline
\end{tabular}

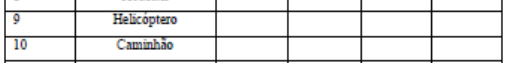

\begin{tabular}{|l|c|l|l|l|l|}
\hline 11 & Caminario & & & & \\
\hline 12 & Bicicleta & & & & \\
\hline 13 & Trem & & & & \\
\hline 14 & Coma & & & & \\
\hline
\end{tabular}

\begin{tabular}{|l|c|l|l|l|l|}
\hline 13 & Cama & & & & \\
\hline 14 & Cadera & & & & \\
\hline 15 & Fogio & & & & \\
\hline
\end{tabular}

\begin{tabular}{|l|l|l}
\hline 15 & Fogio \\
\hline 16
\end{tabular}

\begin{tabular}{|l|c|l|l|l|l|}
\hline 16 & Faca & & & & \\
\hline 17
\end{tabular}
\begin{tabular}{|l|l|l|l|l|l|}
\hline 18 & Boné & & & & \\
\hline 19 & Bonen & & & & \\
\hline
\end{tabular} \begin{tabular}{|l|c|l|l|l|l|}
\hline 19 & Bolsa & & & & \\
\hline 20 & Dentist & & & & \\
\hline 21 & Bombers & & & & \\
\hline
\end{tabular}

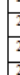

\begin{tabular}{|l|c|l|l|l|l|}
\hline 21 & Bombeiro & & & & \\
\hline 22 & Proféssora & & & & \\
\hline 23 & Igreja & & & & \\
\hline 24 & Predio & & & & \\
\hline 25 & Rotio & & & & \\
\hline 26 & Patins & & & & \\
\hline 27 & Vermelho & & & & \\
\hline 28 & Amarelo & & & & \\
\hline 29 & Qunarrado & & & & \\
\hline 30 & Circulo & & & & \\
\hline \multicolumn{2}{|c|}{ Total } & & & & \\
\hline
\end{tabular}

3. Parte: CONIPLEXIDADE LNGUUISTICA

\begin{tabular}{|c|c|c|c|c|}
\hline Periodos & $\begin{array}{c}\text { Periodo Pré } \\
\text { Linguistico }\end{array}$ & $\begin{array}{c}\text { Estágio } \\
\text { de uma } \\
\text { Palavia }\end{array}$ & $\begin{array}{c}\text { Estágio das } \\
\text { Pimeiras } \\
\text { Combinaçóes }\end{array}$ & $\begin{array}{c}\text { Estágio das } \\
\text { Muiltiplas } \\
\text { Combinaçóes }\end{array}$ \\
\hline LO & & & & \\
\hline LS & & & & \\
\hline
\end{tabular}

FIGURA 3 
Com as alterações propostas, o protocolo original ganha versatilidade em sua aplicação e a possibilidade de indicar informações a respeito do perfil comunicativo da criança surda no que diz respeito à modalidade preferencial e à complexidade do código usado na modalidade exibida na comunicação. A análise do Tipo II também pode sugerir a eficácia do processo de intervenção ao qual a criança se submete, por exemplo, quando se realiza a aplicação do protocolo com a análise do Tipo II antes e depois da aplicação de uma intervenção fonoaudiológica ou educacional específica. O aumento no índice numérico obtido com a média das pontuaçôes neste tipo de análise indicaria que a criança passou a usar recursos mais próximos de língua do que de gestos (em MVE) ou vocalização (em MOA). Obviamente, para esse tipo de comparação, há de se lembrar de outras interferências externas à intervenção aplicada, além do desenvolvimento de um estudo clínico ou educacional criterioso. 